\title{
RESIDUAL HEAT STRESS IMPROVES SPATIAL ROTATION FUNCTION, BUT DOES NOT MODIFY ATTENTION
}

\author{
Margarita Černych, Marius Brazaitis \\ Lithuanian Sports University, Kaunas, Lithuania
}

\begin{abstract}
Background. We aimed to evaluate the effect on cognitive performance (spatial rotation ability, and working memory) after recovery from heat stress when body temperature naturally decreases to initial level.

Methods. Whole body hyperthermia was induced with Finnish sauna bathing. Before (PRE) and 90 min after (POST) heat stress (EXP)/ rest (CON) participants assessed their own overall motivation, level of sleepiness and mood. Also Switching task with a combination of the Manikin (Man) and the Mathematical Processing (Math) test was performed.

Results. Level of sleepiness significantly $(p<.05)$ increased $90 \mathrm{~min}$ after sauna from $1.88 \pm 0.30$ (EXP PRE) to $3.44 \pm 0.45$ (EXP POST), but did not change significantly in CON (PRE $2.81 \pm 0.53$, POST $2.88 \pm 0.30$ ). Participants felt more fatigue $(p<.05)$ during the POST measurements in both trials (CON $3.88 \pm 0.79$ and EXP $5.88 \pm 1.03$ ) compared with before values (CON $2.75 \pm 0.66$ and EXP $2.44 \pm 0.87$ ); 90 min after sauna fatigue was significantly higher $(p<.05)$ in EXP compared with CON. During the EXP POST $(1.49 \pm 0.12 \mathrm{~s})$ measurements response time in Man task significantly decreased $(p<.05)$ compared with PRE $(1.66 \pm 0.16 \mathrm{~s})$, and throughput significantly $(p<.05)$ increased 90 min after sauna (EXP POST $42.09 \pm 3.28$ ) compared with before values (EXP PRE $38.96 \pm 3.31$ ). No other changes were found in Man ant Math task performance.

Conclusion. Residual heat stress increases the level of sleepiness and induces higher feeling of fatigue, but that does not impair attention and concentration. Moreover, residual heat stress improves spatial rotation function.
\end{abstract}

Keywords: heat stress, attention, working memory, fatigue, and sleepiness.

\section{INTRODUCTION}

$\mathrm{S}$ auna bathing may have therapeutic values (Hannuksela \& Ellahham, 2001) and is used for improving beauty and health, or for recovery after exercise (Scoon, Hopkins, Mayhew, $\&$ Cotter, 2007). The effects of heat is mediated via the sympathetic nervous system (Vuori, 1988), and increased internal body temperature is described as a physiological stress (hyperthermia) (Moran, Shitzer, \& Pandolf, 1998), which worsens the function of the cognitive system. Brain temperature increases in parallel with body temperature during heat stress (Nybo, 2012). That may reduce cerebral blood flow due to hypocapnia and following cerebral vasoconstriction (Nelson et al., 2011), as well as dehydration (Trangmar et al., 2014), impair brain metabolism (Nybo, Møller, Volianitis, Nielsen, \& Secher, 2002); and neurophysiological properties (Shibasaki, Namba, Oshiro, Crandall, \& Nakata, 2016; Shibasaki, Namba, Oshiro, Kakigi, \& Nakata, 2017; Yu, Hill, \& McCormick, 2012). Restricted brain blood flow (Shibasaki et al., 2016, 2017) and body liquids loss greater than 2\% (Gopinathan, Pichan, \& Sharma, 1988) could reduce mental performance. Studies with functional imaging techniques demonstrate that during intense emotional states (for example stress) neural activity in some cognitive-processing areas could be suppressed (Drevets \& Raichle, 1998). 
Some studies suggest that the conduction velocity of the ascending somatosensory input (Nakata, Oshiro, Namba, \& Shibasaki, 2015) and reaction time (Shibasaki et al., 2016) is accelerated under increased body temperature or may reduce neural activity in particular brain regions (Shibasaki et al., 2017). Stressors activate the hypothalamopituitary-adrenocortical and the sympatho-adrenal medullary axis, and increase in concentration of cortisol, adrenaline and noradrenaline is observing (McMorris et al., 2006; Minton, 1994). Glucocorticoids cross blood-brain barrier and inflict the cognitive function sustained by the hippocampus and frontal lobes, the two brain regions containing the largest concentration of glucocorticoid receptors (Lupien, Maheu, Tu, Fiocco, \& Schramek, 2007).

Less is known about residual heat stress effect on cognitive function when body temperature naturally recovery till normothermia. A study with a whole body cooling after heat stress (skin temperature elevation $\Delta 4.66 \pm 0.63^{\circ} \mathrm{C}$, esophageal temperature $\Delta 1.30 \pm 0.24^{\circ} \mathrm{C}$ ) involved decrease in error rate (versus heat stress condition) (Shibasaki et al., 2017). (Shibasaki et al., 2016) determined shorter reaction time, but not changed error rate compares with before when whole body cooling after heat stress (esophageal temperature $\Delta$ $2.0^{\circ} \mathrm{C}$ ) was induced. Also cerebral perfusion was eliminated (one of the important factor influencing changes in cognitive performance) in Shibasaki et al. (2016, 2017) studies using face/head cooling. McMorris et al. (2006) determined random movement generation score was significantly poorer comparing pre and post-recovery results (heat stress increased core temperature $1.10 \pm$ $0.11^{\circ} \mathrm{C}$, body core temperature returned to pre heat values during recovery), but no differences in choice reaction time, verbal and spatial recall scores were found, and their participants were not dehydrated. Dehydration results in the increase in the level of plasma cortisol with or without heat stress (Hoffman et al., 1994) and decreases cognitive performance in general (Cian, Barraud, Melin, \& Raphel, 2001). High level of cortisol is an indicator of anxiety and could inhibit cognition (McMorris et al., 2006).

In this study, we aimed to evaluate the effect on cognitive performance (such as spatial rotation ability, attention, concentration and working memory) after recovery from heat stress when body temperature naturally decreases to initial level.
Most of the studies examined changes of cognitive function under heat stress (Hocking, Silberstein, Lau, Stough, \& Roberts, 2001; McMorris et al., 2006; Racinais, Gaoua, \& Grantham, 2008; Razmjou, 1996; Shibasaki et al., 2017) or using different cooling methods after heating (McMorris et al., 2006; Shibasaki et al., 2017). However, we do not use any cool/cold stimuli, which are described as comfortable and increasing alertness (in combination with elevated body temperature (Cabanac, 1971; Nakamura et al., 2008). Medial and orbital regions of prefrontal cortex are connected with the hypothalamus and other limbic structures (Webster, 1982), so psychological perception of recovery might be more important than the physiological one (McMorris et al., 2006). Since more complicated tasks are more vulnerable to stressors (Hocking et al., 2001), we expect that with altered brain homeostasis during heat stress (Shibasaki et al., 2016, 2017), reduced cortical activity after heat exposure (Nybo et al., 2002, Cernych, Satas, \& Brazaitis, 2018) will induce fatigue, impair attention and concentration, and as a result disturbed spatial rotation ability and working memory.

\section{METHODS}

Subjects. Sixteen healthy male volunteers (Table 1) participated in the study. They were considered healthy; with no history of psychiatric or neurological disorder, did not consume any psychotropic substances or other drugs; were physically active, but with no excessive sport activities ( $<3$ times per week); had normal or corrected-to-normal visual activity. Participants were not acclimated to heat. They were asked to avoid intense physical activity for a minimum of $48 \mathrm{~h}$, any food and drink (except water) within $4 \mathrm{~h}$ before testing, and to avoid any eating or drinking during both study trials. The experimental

Table 1. Physical characteristics of the participants

\begin{tabular}{|l|l|}
\hline & Male $(n=16)$ \\
\hline Age $(\mathrm{y})$ & $24 \pm 1$ \\
\hline Height $(\mathrm{m})$ & $84.27 \pm 0.96$ \\
\hline Mass $(\mathrm{kg})$ & $184 \pm 0.52$ \\
\hline BMI $\left(\mathrm{kg} \cdot \mathrm{m}^{-2}\right)$ & $24.73 \pm 0.20$ \\
\hline Body fat $(\%)$ & $15.74 \pm 1.25$ \\
\hline
\end{tabular}

Note. Values are shown as the mean \pm SEM. 
procedures were approved by the Lithuanian University of Health Sciences Kaunas Region Biomedical Research Ethics Committee.

Experimental protocol. The experiment was designed to investigate post-sauna residual effects on directed attention and executive function in addition to the abilities evaluated by the individual tests (i.e., visuo-spatial skills and mathematical computation). The subjects were familiarized with the procedure 1 week before the trial and practiced switching task three times on three different days. In an experimental (EXP) trial, on arrival at the laboratory each subject was asked to rest in a semi recumbent posture for $20 \mathrm{~min}$ in a thermally neutral ambient temperature $\left(\mathrm{Ta} 23^{\circ} \mathrm{C}\right)$. Rectal temperature $\left(\mathrm{T}_{\mathrm{re}}\right)$, stabilized heart rate (HR) and subjective ratings were recorded. After resting measurements, the participants seated at a table with a computer and were asked to fill motivation, sleepiness and mood scales and to perform switching task. Upon competition of the task, the subjects were asked to enter a sauna. Sauna $\left(T_{a} 80-90 o C\right.$, rh $20 \%$ ) bathing consisted of four sets (first one $15 \mathrm{~min}$, next three sets $10 \mathrm{~min}$, with 15 rest between each set with the subject in a semirecumbent posture at $\mathrm{T}_{\mathrm{a}} 23^{\circ} \mathrm{C}$ ).

Before the end of the fourth sauna set, the participants' $T_{r e}$, HR and subjective ratings were recorded. After sauna bathing, participants took a warm shower and rested in Ta $23^{\circ} \mathrm{C}$ for 90 min until $\mathrm{T}_{\mathrm{re}}$ and HR recovered till preheating level. $90 \mathrm{~min}$ after sauna bathing participants were asked to fill in motivation, sleepiness and mood scales and to perform switching task. In the control (CON) case, instead of sauna the subject spent time in thermal neutral environment. The order of trials for each participant was random.

Measurements of core body temperature and cardiovascular responses. The $T_{r e}$ was measured using a rectal thermocouple (Rectal Probe, Ellab, Hvidovre, Denmark; accuracy \pm $0.01^{\circ} \mathrm{C}$ ), which was inserted by each participant 12 $\mathrm{cm}$ past the anal sphincter. Consecutive $5 \mathrm{~s}$ average HR was recorder (S-625X, Polar Electro, Kempele, Finland). Physiological strain index (PSI) was calculated using equation

PSI $=5\left(\mathrm{~T}_{\text {ret }}-\mathrm{T}_{\text {re } 0}\right) \times\left(39.5-\mathrm{T}_{\text {re0 }}\right)^{-1}+5\left(\mathrm{HR}_{\mathrm{t}}-\mathrm{HR}_{0}\right) \times$ $\left(180-\mathrm{HR}_{0}\right)^{-1}$

The $\mathrm{T}_{\text {re0 }}$ and $\mathrm{HR}_{0}$ measurements were taken before sauna; $\mathrm{T}_{\text {ret }}$ and $\mathrm{HR}_{\mathrm{t}}$ measurements were taken before leaving the sauna (Moran et al., 1998), where PSI range from 1 (no heat stress) to 10 (very high heat stress) within the limits of the following values: $36.5 \leq \mathrm{T}_{\mathrm{re}} \leq 39.5^{\circ} \mathrm{C}$ and $60 \leq \mathrm{HR} \leq 180$ beats $\min ^{-1}$.

Subjective ratings. The method was described by Ha, Tokura, Tanaka, and Holmer (1996) and adapted by Cernych et al. (2017) was used to evaluate subjective ratings for thermal sensations, shivering/sweating and thermal comfort. Thermal sensation ratings ranged from 1 (very cold) to 9 (very hot), with 5 being neutral. Shivering/sweating ratings ranged from 1 (heavily sweating) to 7 (vigorous shivering), with 4 being neutral. Thermal comfort ratings ranged from 1 (comfortable) to 5 (especially uncomfortable). Subjective ratings were recorded in the end of sauna bathing to evaluate sauna induced subjective level of stress.

Scales. Participants assessed their own overall motivation rating using the scale from 0 (not at all) to 4 (extremely). Level of sleepiness was evaluated using scale from 1 (vigor, viable, awake) to 8 (like sleeping). Mood was assessed using the Brunel mood scale (BRUMS), which contains 24 simple mood descriptors. Participants indicated whether they experienced such feelings on a 5-point scale ( 0 - not at all, 4 - extremely). The 24 items comprised the following 6 subscales: anger, confusion, depression, fatigue, tension and vigor, subscale score in range from 0 to 16 .

Cognitive domain. Switching task was used from Automated Neuropsychological Assessment Metrics (ANAM4 ${ }^{\mathrm{TM}}$ ). The task is a combination of the Manikin (Man) test and the Mathematical Processing (Math) test. The man (Manikin part) is holding a ball in one hand and a cube in the other hand, and a ball or a cube is displayed at the bottom of the screen. The man is standing upright or upside down and either facing toward the user or facing away. The user is instructed to determine which of the man's hands is holding the object displayed at the bottom of the screen and to press one designated " $\mathrm{W}$ " to indicate the left hand and "D" to indicate the right hand.

During Mathematical Processing task, an arithmetic problem involving three single-digit numbers and two operators is displayed (e.g., "5 $2+3=$ "). The user presses " $\mathrm{J}$ " buttons to indicate whether the answer to the problem is less than five or "I" button when answer is greater than five. One problem from each test appears on the display. The problems appear simultaneously side-by-side, 
and the participant is directed by means of a red arrow at the bottom of the screen to respond to the problem on the left or on the right. Responses are entered using the keyboard as in the individual tests, with the left hand used for the Manikin and the right hand used for Mathematical Processing. In a variation of the test, only one type of problem is presented on each display and the user responds as appropriate for the given test. For each task accuracy (in \%), response time (RT, in s) for correct response and throughput were assessed. Throughput was calculated using an equation:

Throughput $=[$ NumCorr $/($ NumCorr + NumInc $) *$ MeanRT + NumLapse * Timeout)],

where NumCorr - number of correct responses, NumInc - number of incorrect responses, MeanRT - mean response time for all items (correct and incorrect), NumLapse - number of trials where no response was made in the allotted time, Timeout - allotted time.

Statistical analysis. The data were tested for normal distribution using the KolmogorovSmirnov test, and all were found to be normally distributed. The data are presented as mean and standard deviation (SD). A two-way analysis ANOVA (General Linear Model) was used to determine the effects of residual effects of sauna bathing on cognitive performance (before vs $90 \mathrm{~min}$ after) as within-subject factor of two levels and trial (CON vs EXP) as within-subject factor of two levels. If significant effects were found, Tukey's post-hoc adjustment was used for multiple comparisons within each repeated-measure ANOVA. A dependent-sample t-test was used to localize the time point difference. Nonparametric related samples test was applied to evaluate changes in motivation, level of sleepiness and mood.

\section{RESULTS}

Before leaving the sauna participants experienced between moderate and high stress (Table 2), felt between hot and very hot, were between moderate and heavily sweating and felt uncomfortable. Overall motivation did not change before and after sauna, as well as in CON. Level of sleepiness significantly $(p<.05)$ increased $90 \mathrm{~min}$ after sauna from $1.88 \pm 0.30$ (EXP PRE) to $3.44 \pm$ 0.45 (EXP POST), but did not change significantly in CON (PRE $2.81 \pm 0.53$, POST $2.88 \pm 0.30$, Figure 1).
Table 2. Physiological strain index (PSI) and subjective ratings at the end of sauna bathing (before leaving the sauna)

\begin{tabular}{|l|c|}
\hline & Male $(\mathbf{n}=\mathbf{1 6})$ \\
\hline PSI & $6.45 \pm 1.47$ \\
\hline Thermal sensation & $8.38 \pm 0.18$ \\
\hline Sweating & $1.97 \pm 0.19$ \\
\hline Thermal comfort & $3.00 \pm 0.29$ \\
\hline
\end{tabular}

Note. Values are shown as the mean $\pm \mathrm{S}$.

Figure 1. Subjective evaluation of motivation and level of sleepiness before (PRE) and 90 min after (POST) sauna (EXP)/ rest (CON)

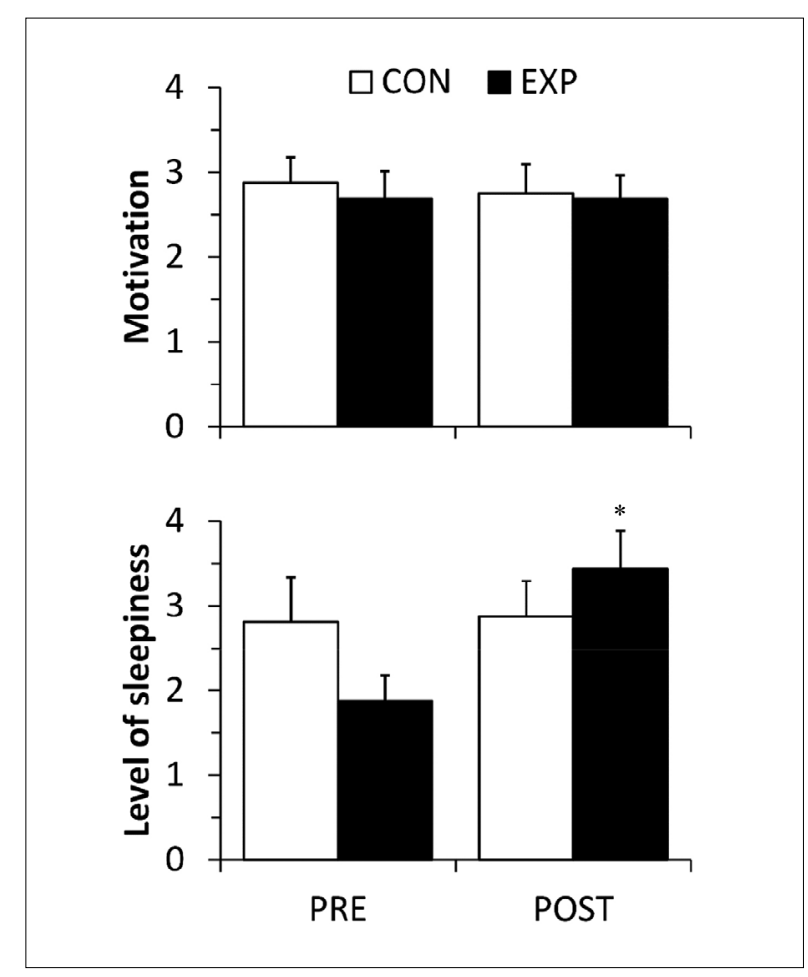

Notes. Values are shown as the Mean $\pm S D . * p<.05$ compared with values before.

Anger (CON PRE $0.13 \pm 0.34$, CON POST $0.21 \pm 0.40$, EXP PRE $0.06 \pm 0.25$, EXP POST $0.12 \pm 0.33$ ), confusion (CON PRE $0.38 \pm 0.72$, CON POST $0.19 \pm 0.39$, EXP PRE $0.06 \pm 0.25$, EXP POST $0.02 \pm 0.08)$, depression ( 0 in all cases) and tension (CON PRE $0.19 \pm 0.40$, CON POST $0.19 \pm 0.54$, EXP PRE $0.31 \pm 0.70$, EXP POST $0.13 \pm 0.34)$ did not change significantly comparing before values and those $90 \mathrm{~min}$ after in both trials (CON and EXP). Participants felt more fatigue $(p<.05)$ during the POST measurements in both trials (CON $3.88 \pm 0.79$ and EXP $5.88 \pm 1.03$ ) compared with before values (CON $2.75 \pm 0.66$ and EXP $2.44 \pm 0.87)$; 90 min after sauna fatigue was 
Figure 2. Subjective evaluation of fatigue and vigor before (PRE) and 90 min after (POST) sauna (EXP)/ rest (CON)

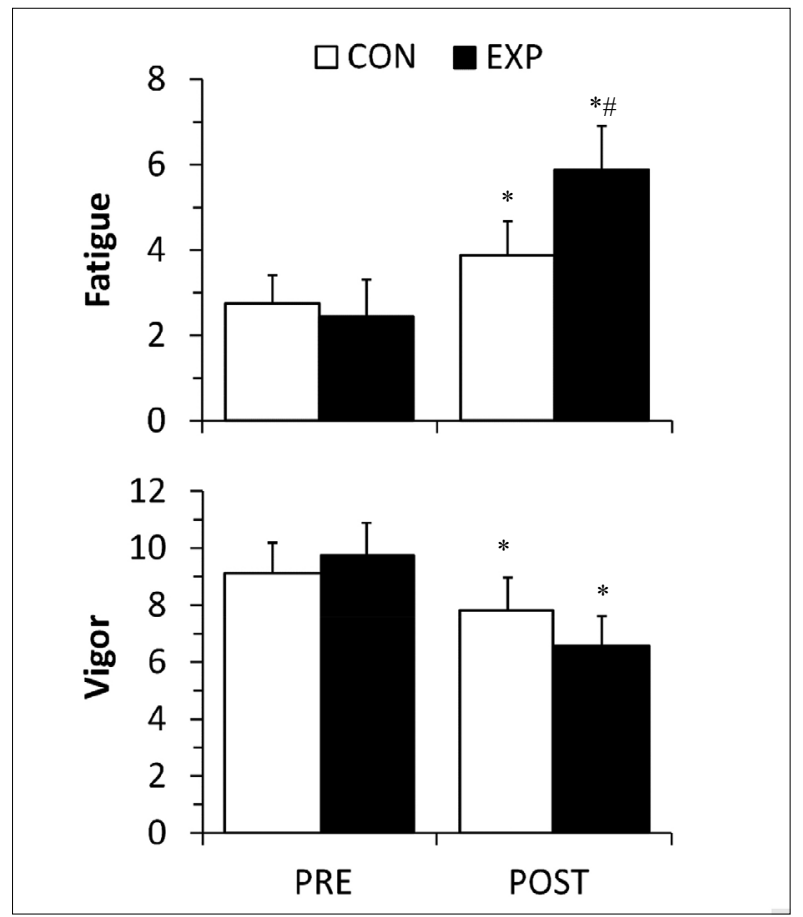

Notes. Values are shown as the Mean $\pm S D .{ }^{*} p<.05$ compared with values before, $\# p<.05$ compared with $\mathrm{CON}$. significantly higher $(p<.05)$ in EXP compared with CON (Figure 2).

No changes in CON were found in both tasks (Man and Math; RT, accuracy and throughput), also no changes in Math task (RT, accuracy and throughput) in EXP were found comparing PRE and POST measurements (Figure 3). During the EXP POST $(1.49 \pm 0.12 \mathrm{~s})$ measurements, RT in Man task significantly decreased $(p<.05)$ compared with PRE $(1.66 \pm 0.16 \mathrm{~s})$, and throughput significantly $(p<.05)$ increased 90 min after sauna (EXP POST $42.09 \pm 3.28$ ) compared with before (EXP PRE $38.96 \pm 3.31$ ). No significant changes in accuracy were found in EXP Man task.

\section{DISCUSSION}

Functional neuroimaging studies show the potential to elucidate neural mechanism underlying the complex interaction between emotion and cognition, when brain regions involved in performing
Figure 3. The manikin (Man) and the Mathematical Processing (Math) test mean response time (s), mean percentage (accuracy, $\%)$ and throughput of correct responses before (PRE) and 90 min after (POST) sauna (EXP)/ rest $(\mathrm{CON})$
Notes. Values are shown as the Mean $\pm S D$. $* p<.05$ compared with values before.

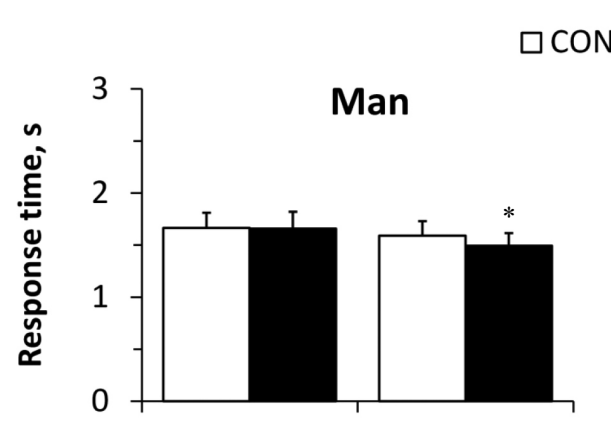

EXP
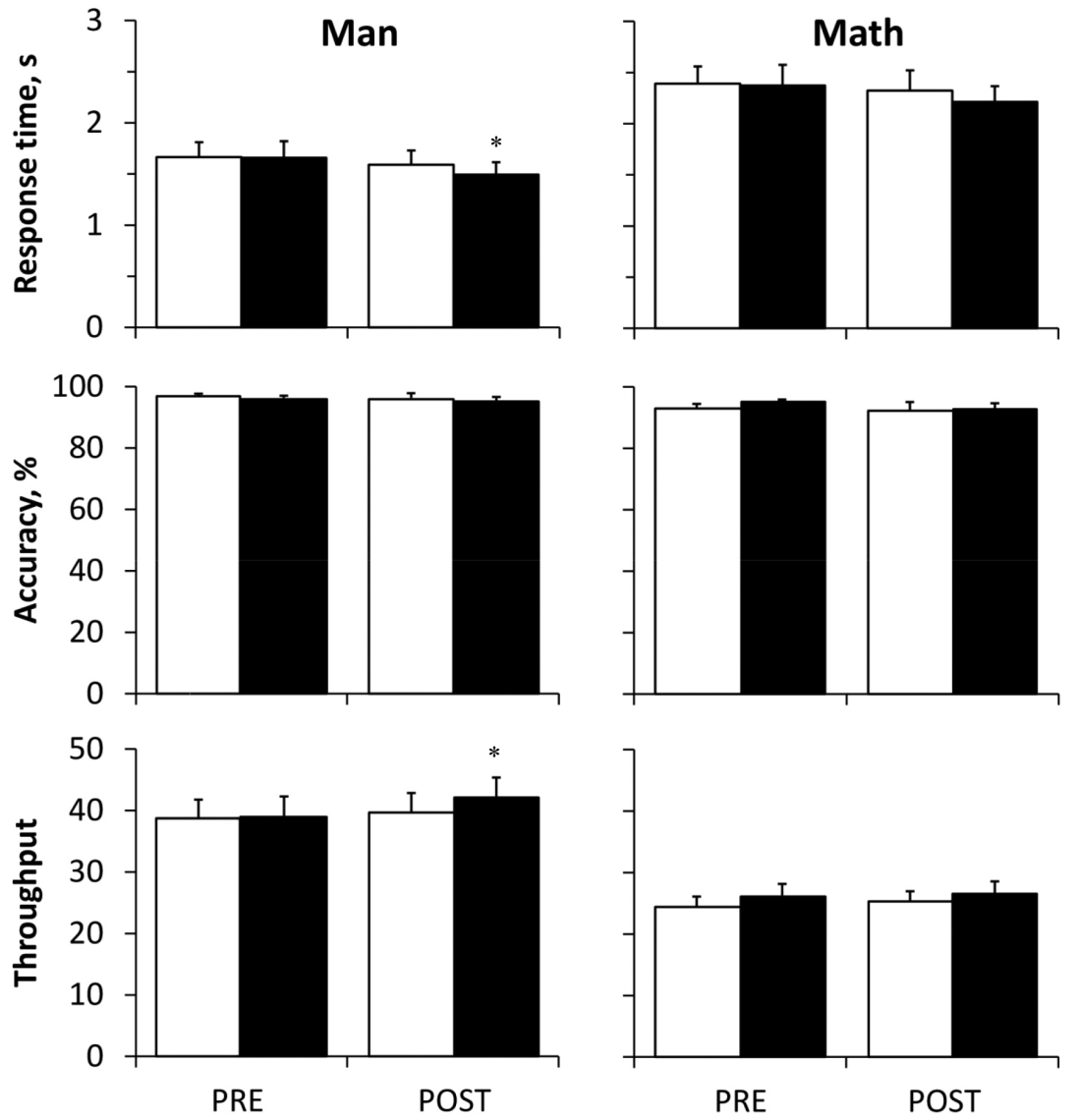
some memory or visuospatial tasks become activated, brain regions involved in emotional processing become less active (Drevets \& Raichle, 1998). Passive heat exposure which perturbs deep body (and brain) temperature could impair vigilance (Hancock, 1986), damage pre-attentive processing in central nervous system level for a long time (Sun, Li, Min, \& Qingjun, 2011), decrease working memory capacity as a result of frontal lobe activity dysfunction in hyperthermia conditions (Racinais et al., 2008). Still the present study shows that post heat recovery induced higher level of sleepiness and higher subjective rating of fatigue feeling, but performance of Math task stays in similar level and results (RT and throughput) of Man task even improve 90 min after heat exposure.

A general consensus that heat exposure is modifying cognition, but the level of performance changes is dependent on the severity of heat strain and the complexity of the task (Hocking et al., 2001). Hyperthermia can improve cognitive performance if the increase in temperature does not disturb homeostasis (Hancock, 1986) and improvement could be due to an activation of the thermoregulatory mechanisms and general physiological arousal (Teichner, 1966). Cognitive function can be improved by moderate exercise (Kamijo et al., 2004; Lambourne, \& Tomporowski, 2010). It seems like moderate stress could have positive effect on cognition.

McMorris et al. (2006) showed decreased vigour with no significant changes in reaction time, verbal recall and spatial recall scores after post-recovery. No changes in cognitive performance accompanied with no changes in adrenaline, noradrenaline and serotonin. Only decrease in cortisol was evaluated, but that probably due to cortisol circadian rhythm (McMorris et al., 2006). It seem like rehydrated participant were almost fully recovered in 90 min after heat exposure. As mentioned before, dehydration has significant influence on level of plasma cortisol (Hoffman et al., 1994). Cancelation of this factor might have significant influence on results. In another study participants with recovered cerebral perfusion after heat exposure showed shorter reaction time in auditory oddball task (Shibasaki et al., 2016). Rasmussen, Stie, Nybo, \& Nielsen (2004) concluded that reduced alertness during hyperthermia was unrelated with decreased cerebral blood flow. Thus, one of the reasons for improved reaction time could be cold stimuli. When body temperature is high, cold or cool stimuli to the body surface are perceived as being comfortable and increase alertness (Cabanac, 1971; Nakamura et al., 2008). Also participant in Shibasaki et al.s' ( 2016) study were dehydrated as in the present study. Cortisol could modify mood and cognition (McMorris et al., 2006). The level of cortisol reaches its peak $30 \mathrm{~min}$ after stress and remains elevated $1 \mathrm{~h}$ after (Skurvydas et al., 2017), in combination with dehydration, and it is possible that $90 \mathrm{~min}$ it still remains elevated. Despite higher fatigue and sleepiness levels, moderately elevated level of cortisol can have positive effect on cognition. Whereas $90 \mathrm{~min}$ after heats stress termination participants partly recovered from stress, due to slow cortisol recovery till baseline (Skurvydas et al., 2017) and dehydration (Hoffman et al., 1994), so cortisol level probably remains elevated, but not to the peak value. Moderate elevation of stress hormone could improve cognitive performance.

Changesinbrain temperaturemay alterneuronal activity, brain metabolism, and cerebral blood flow (Wang et al., 2014). Even small temperature gradients may directly modulate presynaptic and postsynaptic events (Fuxe et al., 2005). Studies with mammals showed an approximately $10 \mathrm{C}$ gradient exists between the cooler cortical region and warmer basal regions (Hayward \& Baker, 1969; Serota \& Gerard, 1938), also regional differences in the increase in brain temperature during dynamic exercise were observed (Wang et al., 2014). The left presubiculum, retrosplenial complex, and parietal-occipital sulcus coded location identity (Vass \& Epstein, 2013). Working memory activates the fronto-parietal brain regions, including the prefrontal, cingulate, and parietal cortices (Chai, Abd Hamid, \& Abdullah, 2018). Performing Man and Math tasks different brain regions are activated. However in brain exist regional temperature differences (Hayward \& Baker, 1969; Serota \& Gerard, 1938), and blood-brain barrier, so permeability, is temperature dependent (Kiyatkin, 2005) we speculatively assume, that hyperthermia differently influence spatial rotation ability and working memory pathways.

\section{CONCLUSION}

Residual heat stress increases the level of sleepiness and induces higher feeling of fatigue, but that does not impair attention and concentration. Moreover, residual heat stress improves spatial rotation function. 


\section{REFERENCES}

Cabanac, M. (1971). Physiological role of pleasure. Science, 173(4002), 1103-1107. https://doi.org/10.1126/ science. 173.4002 .1103

Cernych, M., Baranauskiene, N., Eimantas, N., Kamandulis, S., Daniuseviciute, L., \& Brazaitis, M. (2017). Physiological and psychological responses during exercise and recovery in a cold environment is gender-related rather than fabric-related. Frontiers in Psychology, 8. https://doi.org/10.3389/fpsyg.2017.01344

Cernych, M., Satas, A., \& Brazaitis. M. (2018). Postsauna recovery enhances brain neural network relaxation and improves cognitive economy in oddball tasks. International Journal of Hyperthermia. https://doi.org/ 10.1080/02656736.2018.1504992 (in press)

Chai, W. J., Abd Hamid, A. I., \& Abdullah, J. M. (2018). Working memory from the psychological and neurosciences perspectives: A review. Frontiers in Psychology, 9, 401. https://doi.org/10.3389/ fpsyg.2018.00401

Cian, C., Barraud, P. A., Melin, B., \& Raphel, C. (2001). Effects of fluid ingestion on cognitive function after heat stress or exercise-induced dehydration. International Journal of Psychophysiology, 42(3), 243-251. https:// doi.org/10.1016/S0167-8760(01)00142-8

Drevets, W. C., \& Raichle, M. E. (1998). Suppression of regional cerebral blood during emotional versus higher cognitive implications for interactions between emotion and cognition. Cognition and Emotion, 12(3), 353-385. https://doi.org/10.1080/026999398379646

Fuxe, K., Rivera, A., Jacobsen, K. X., Höistad, M., Leo, G., Horvath, T. L., ... Agnati, L. F. (2005). Dynamics of volume transmission in the brain. Focus on catecholamine and opioid peptide communication and the role of uncoupling protein 2. Journal of Neural Transmission (Vienna, Austria: 1996), 112(1), 65-76. https://doi.org/10.1007/s00702-004-0158-3

Gopinathan, P. M., Pichan, G., \& Sharma, V. M. (1988). Role of dehydration in heat stress-induced variations in mental performance. Archives of Environmental Health, 43(1), 15-17.

Ha, M., Tokura, H., Tanaka, Y., \& Holmer, I. (1996). Effects of Two kinds of underwear on thermophysiological responses and clothing microclimate during $30 \mathrm{~min}$ walking and $60 \mathrm{~min}$ recovery in the cold. Applied Human Science, 15(1), 33-39. https://doi.org/10.2114/jpa.15.33 Hancock, P. A. (1986). Sustained attention under thermal stress. Psychological Bulletin, 99(2), 263-281.

Hannuksela, M. L., \& Ellahham, S. (2001). Benefits and risks of sauna bathing. The American Journal of Medicine, 110(2), 118-126. https://doi.org/10.1016/ S0002-9343(00)00671-9

Hayward, J. N., \& Baker, M. A. (1969). A comparative study of the role of the cerebral arterial blood in the regulation of brain temperature in five mammals. Brain Research, 16(2), 417-440.
Hocking, C., Silberstein, R. B., Lau, W. M., Stough, C., \& Roberts, W. (2001). Evaluation of cognitive performance in the heat by functional brain imaging and psychometric testing. Comparative Biochemistry and Physiology Part A: Molecular \& Integrative Physiology, 128(4), 719734. https://doi.org/10.1016/S1095-6433(01)00278-1

Hoffman, J. R., Maresh, C. M., Armstrong, L. E., Gabaree, C. L., Bergeron, M. F., Kenefick, R. W., ... Ward, A. (1994). Effects of hydration state on plasma testosterone, cortisol and catecholamine concentrations before and during mild exercise at elevated temperature. European Journal of Applied Physiology and Occupational Physiology, 69(4), 294-300.

Kamijo, K., Nishihira, Y., Hatta, A., Kaneda, T., Wasaka, T., Kida, T., \& Kuroiwa, K. (2004). Differential influences of exercise intensity on information processing in the central nervous system. European Journal of Applied Physiology, 92(3), 305-311. https:// doi.org/10.1007/s00421-004-1097-2

Kiyatkin, E. A. (2005). Brain hyperthermia as physiological and pathological phenomena. Brain Research Reviews, 50(1), 27-56. https://doi.org/10.1016/j.brainresrev.2005.04.001

Lambourne, K., \& Tomporowski, P. (2010). The effect of exercise-induced arousal on cognitive task performance: A meta-regression analysis. Brain Research, 1341, 1224. https://doi.org/10.1016/j.brainres.2010.03.091

Lupien, S. J., Maheu, F., Tu, M., Fiocco, A., \& Schramek, T. E. (2007). The effects of stress and stress hormones on human cognition: Implications for the field of brain and cognition. Brain and Cognition, 65(3), 209-237. https://doi.org/10.1016/j.bandc.2007.02.007

McMorris, T., Swain, J., Smith, M., Corbett, J., Delves, S., Sale, C., ... Potter, J. (2006). Heat stress, plasma concentrations of adrenaline, noradrenaline, 5-hydroxytryptamine and cortisol, mood state and cognitive performance. International Journal of Psychophysiology, 61(2), 204215. https://doi.org/10.1016/j.ijpsycho.2005.10.002

Minton, J. E. (1994). Function of the hypothalamicpituitary-adrenal axis and the sympathetic nervous system in models of acute stress in domestic farm animals. Journal of Animal Science, 72(7), 1891-1898. https://doi.org/10.2527/1994.7271891x

Moran, D. S., Shitzer, A., \& Pandolf, K. B. (1998). A physiological strain index to evaluate heat stress. American Journal of Physiology - Regulatory, Integrative and Comparative Physiology, 275(1), R129-R134.

Nakamura, M., Yoda, T., Crawshaw, L. I., Yasuhara, S., Saito, Y., Kasuga, M., ... Kanosue, K. (2008). Regional differences in temperature sensation and thermal comfort in humans. Journal of Applied Physiology (Bethesda, Md.: 1985), 105(6), 1897-1906. https://doi.org/10.1152/ japplphysiol.90466.2008

Nakata, H., Oshiro, M., Namba, M., \& Shibasaki, M. (2015). Effects of passive heat stress on human 
somatosensory processing. American Journal of Physiology - Regulatory, Integrative and Comparative Physiology, 309(11), R1387-R1396. https://doi. org/10.1152/ajpregu.00280.2015

Nelson, M. D., Haykowsky, M. J., Stickland, M. K., Altamirano-Diaz, L. A., Willie, C. K., Smith, K. J., ... Ainslie, P. N. (2011). Reductions in cerebral blood flow during passive heat stress in humans: Partitioning the mechanisms. The Journal of Physiology, 589(16), 40534064. https://doi.org/10.1113/jphysiol.2011.212118

Nybo, L. (2012). Brain temperature and exercise performance. Experimental Physiology, 97(3), 333-339. https://doi.org/10.1113/expphysiol.2011.062273

Nybo, L., Møller, K., Volianitis, S., Nielsen, N., \& Secher, N. H. (2002). Effects of hyperthermia on cerebral blood flow and metabolism during prolonged exercise in humans. Journal of Applied Physiology, 93(1), 58-64. https://doi.org/10.1152/japplphysiol.00049.2002

Racinais, S., Gaoua, N., \& Grantham, J. (2008). Hyperthermia impairs short-term memory and peripheral motor drive transmission. The Journal of Physiology, 586(19), 4751-4762. https://doi.org/10.1113/jphysiol.2008.157420

Rasmussen, P., Stie, H., Nybo, L., \& Nielsen, B. (2004). Heat induced fatigue and changes of the EEG is not related to reduced perfusion of the brain during prolonged exercise in humans. Journal of Thermal Biology, 29(7), 731-737. https://doi.org/10.1016/j.jtherbio.2004.08.047

Razmjou, S. (1996). Mental workload in heat: Toward a framework for analyses of stress states. Aviation, Space, and Environmental Medicine, 67(6), 530-538.

Scoon, G. S. M., Hopkins, W. G., Mayhew, S., \& Cotter, J. D. (2007). Effect of post-exercise sauna bathing on the endurance performance of competitive male runners. Journal of Science and Medicine in Sport, 10(4), 259-262. https://doi.org/10.1016/j.jsams.2006.06.009

Serota, H. M., \& Gerard, R. W. (1938). Localized thermak changes in the cat's brain. Journal of Neurophysiology, 1(2), 115-124. https://doi.org/10.1152/jn.1938.1.2.115

Shibasaki, M., Namba, M., Oshiro, M., Crandall, C. G., \& Nakata, H. (2016). The effect of elevations in internal temperature on event-related potentials during a simple cognitive task in humans. American Journal of Physiology - Regulatory, Integrative and Comparative Physiology, 311(1), R33-R38. https://doi.org/10.1152/ ajpregu.00086.2016
Shibasaki, M., Namba, M., Oshiro, M., Kakigi, R., \& Nakata, H. (2017). Suppression of cognitive function in hyperthermia; From the viewpoint of executive and inhibitive cognitive processing. Scientific Reports, 7, 43528. https://doi.org/10.1038/srep43528

Skurvydas, A., Verbickas, V., Eimantas, N., Baranauskiene, N., Cernych, M., Skrodeniene, E., ... Brazaitis, M. (2017). Psychological and physiological biomarkers of neuromuscular fatigue after two bouts of sprint interval exercise. Frontiers in Psychology, 8, 2282. https://doi.org/10.3389/fpsyg.2017.02282

Sun, G., Li, L., Min, L., \& Qingjun, J. (2011). Hyperthermia impaired pre-attentive processing: An auditory MMN study - ScienceDirect. Neuroscience Letters, 502(2), 94-98. https://doi.org/10.1016/j. neulet.2011.07.029

Teichner, W. H. (1966). Individual thermal and behavioral factors in cold-induced vasodilation. Psychophysiology, 2(4), 295-304. https://doi. org/10.1111/j.1469-8986.1966.tb02657.x

Trangmar, S. J., Chiesa, S. T., Stock, C. G., Kalsi, K. K., Secher, N.H., \& González-Alonso, J.(2014). Dehydration affects cerebral blood flow but not its metabolic rate for oxygen during maximal exercise in trained humans. The Journal of Physiology, 592(14), 3143-3160. https://doi. org/10.1113/jphysiol.2014.272104

Vass, L. K., \& Epstein, R. A. (2013). Abstract representations of location and facing direction in the human brain. The Journal of Neuroscience: The Official Journal of the Society for Neuroscience, 33(14), 6133-6142. https://doi.org/10.1523/ JNEUROSCI.3873-12.2013

Vuori, I. (1988). Sauna bather's circulation. Annals of Clinical Research, 20(4), 249-256.

Wang, H., Wang, B., Normoyle, K. P., Jackson, K., Spitler, K., Sharrock, M. F., ... Du, R. (2014). Brain temperature and its fundamental properties: A review for clinical neuroscientists. Frontiers in Neuroscience, 8. https://doi.org/10.3389/fnins.2014.00307

Webster, K. E. (1982). The Prefrontal cortex: Anatomy, physiology and neuropsychology of the frontal lobe. Journal of Anatomy, 134(Pt 3), 592.

Yu, Y., Hill, A. P., \& McCormick, D. A. (2012). Warm body temperature facilitates energy efficient cortical action potentials. PLOS Computational Biology, 8(4), e1002456. https://doi.org/10.1371/journal.pcbi.1002456 